\title{
Are Cosmic Rays still Useful?
}

IT is not surprising in some ways that cosmic-ray physics is regarded by some people as the poor relation of accelerator physics. It has often happened, indeed, that cosmic-ray experiments have not been as successful as they might have been, not because they were badly designed but because they have been built too cheaply and sometimes on too small a scale.

In the euphoria which has surrounded the building and successful operation of large accelerators during the past fifteen or twenty years, many people seem to have lost sight of the fact that accelerators-even the new generation which will quite soon become operationalcan only produce particles with energies of a few hundred $\mathrm{GeV}$, albeit with a reasonable intensity. But some of the particles which impinge on the Earth's atmosphere (and some of those produced by interactions within the atmosphere itself) have energies many orders of magnitude greater than those available at accelerators.

But this is not to say, of course, that cosmic rays are the ideal source of energetic charged particles. This is particularly true of the very high energy flux which is so small that it requires very large apparatus for its meaningful study. Nonetheless, some accelerator physicists are realizing that cosmic-ray experiments can profitably be carried out at accelerator laboratories. Professor Kasha at Brookhaven, for example, is using a large solid-iron magnet spectrometer to measure the sea level momentum spectrum of cosmic-ray muons. The spectrum reflects the nature of the interactions of primary cosmic rays, which are known to have energies as high as $10^{19} \mathrm{eV}$ at the top of the atmosphere, and is, for this reason, very worthy of study by physicists interested in elementary particle interactions.

Not all useful cosmic-ray physics needs large quantities of expensive equipment, however. In the past decade or so, momentum spectrum measurements have been made principally with solid iron magnets but, at energies greater than about $1 \mathrm{TeV}$, instruments of this type are not only expensive but cumbersome and difficult to build. Other methods are being increasingly used at these energies, even though they are not as accurate. Instead of calculating the momentum of a muon directly from its deflexion in a magnetic field, this value can be inferred-for example from the interactions which the muon undergoes.

Just such an experiment has been performed by a group from Osaka City, Kobe and Okayama Universities in Japan (Chin et al., Nuovo Cimento B, 4, 177 ; 1971). The essential feature of this sort of measurement is that it should be carried out underground so that the products of muon interactions in the rock above the apparatus can be examined. But the depth must not be so large that the natural sea level muon flux is seriously modified nor so small that the muons are accompanied by strongly interacting particles. The Japanese apparatus is, in principle, very simple; it comprises two plastic scintillation counters which are operated in a railway tunnel under $40 \mathrm{~m}$ water equivalent of rock. Muons passing through the rock produce large cascade showers of electrons (the principal source of energy loss) and if the showers are sufficiently large and energetic they are easily detected.

The cascade showers can be generated by several processes and it is partly because of this that the measure- ments are so useful; a theoretical energy spectrum based on the known parameters of the interactions can be compared with the measured spectrum at very high energies to find out whether or not the interaction cross sections are as expected at these energies.

Of the four interactions which give rise to cascade showers three are electromagnetic in nature and the fourth is nuclear in the sense that it involves the interaction of the virtual photons of the muon with a nucleon to produce strongly interacting particles. Some of these (the uncharged pions) will decay rapidly into a pair of gamma rays which will in turn produce a cascade shower by the materialization process.

The critical parameter of these bursts, as they are called, is the number of ionizing particles at a given level. Chin et al. extracted this information from the scintillators using two sets of twenty photomultiplier tubes which each detected the light from $1 \mathrm{~m}^{2}$ of the two scintillator sheets. When the scintillator signals were compared, the core of the cascade shower could be identified and the number of electrons and positrons in the shower deduced; those containing less than ten particles are of little interest, however, because they are chiefly produced by muons with momenta that can already be studied by other means.

A series of analytical steps has allowed Chin et al. to deduce the energy spectrum of the muons from the burst size spectrum. To justify both their experimental approach and the methods of analysis adopted they have made careful comparisons with the spectra measured by other workers at momenta up to $1 \mathrm{TeV} / \mathrm{c}$. Although their spectrum agrees very closely with other spectra at energies of about $100 \mathrm{GeV}$ (for example, that of Menon and Ramana Murthy, Prog. Elementary Particle and Cosmic Ray Physics, 9, 164 ; 1967), there is some discrepancy at between 1 and $8 \mathrm{TeV}$. The spectrum deduced by Menon and Ramana Murthy has an integral spectral exponent of 2.9 compared with the value of 2.5 suggested by the results of Chin et al. But when the errors associated with the two spectra are fully taken into account, there seems to be very little difference between them.

Chin et al. point out, however, that if the difference between the two spectra turns out to be real in spite of the agreement within the limits of experimental error, there are certain important consequences for the energy loss of high energy muons. In particular, the thickness of matter through which a muon of a given energy will pass before it stops would seem to be smaller according to the Japanese results. This suggests that the energy losses of muons with energies of a few $\mathrm{TeV}$ are greater than expected. Chin et al. conjecture that the cross section for the muon nuclear interaction process-the least well known experimentally because of its small value-is larger than predicted by electromagnetic theories. If this is the case, it would be an interesting confirmation of the somewhat contentious results of a cloud chamber experiment performed about ten years ago by Higashi et al. (Nuovo Cimento, 22, 304 ; 1961). Some of the muon nuclear interactions photographed in this experiment seemed to have energy transfers that were very much larger than theory would predict. There is now an added incentive for other cosmic-ray physicists to design experiments to clarify the matter. 